\title{
Arenobufagin activates p53 to trigger esophageal squamous cell carcinoma cell apoptosis in vitro and in vivo
}

\author{
Junhong Lv' \\ Shaohuan Lin' \\ Panli Peng ${ }^{2}$ \\ Changqing $\mathrm{Cai}^{2}$ \\ Jianming Deng' \\ Mingzhi Wang' \\ Xuejun $\mathrm{Li}^{\prime}$ \\ Rongsheng $\operatorname{Lin}^{3}$ \\ Yu Lin ${ }^{4}$ \\ Ailing Fang 5 \\ Qiling $\mathrm{Li}^{5}$
}

'Thoracic Surgeons Department, 2Oncology No 2 Department, Guangdong No 2 Provincial People's Hospital, Guangzhou, ${ }^{3}$ Department of Oncology, Shunde Longjiang Hospital, Foshan, ${ }^{4}$ Department of Gastroenterology, Puning Overseas Chinese Hospital, ${ }^{5} \mathrm{Galactophore}$ Department, Puning Maternity and Child Care Hospital, Puning, People's Republic of China
Correspondence: Shaohuan Lin Thoracic Surgeons Department, Guangdong No 2 Provincial People's Hospital, Haizhuqu District Xingang Road No 466, Building 7, No 3 Compound, Guangzhou 510317, People's Republic of China Email liziepan@I63.com
This article was published in the following Dove Press journal:

OncoTargets and Therapy

28 February 2017

Number of times this article has been viewed
Abstract: Esophageal squamous cell carcinoma (ESCC) is often diagnosed at late incurable stage and lacks effective treatment strategy. Bufadienolides are cardiotonic steroids isolated from the skin and parotid venom glands of the toad Bufo bufo gargarizans Cantor with novel anticancer activity. However, there is little information about the effects and action mechanisms of bufadienolides on ESCC cells. In this study, the in vitro and in vivo anti-ESCC activities of bufadienolides, including bufalin $(\mathrm{Bu})$ and arenobufagin $(\mathrm{ArBu})$, were examined and the underlying molecular mechanisms were elucidated. The results showed that $\mathrm{ArBu}$ exhibited higher anticancer efficacy than $\mathrm{Bu}$ against a panel of five ESCC cells, with $\mathrm{IC}_{50}$ values ranging from $0.8 \mu \mathrm{M}$ to $3.6 \mu \mathrm{M}$. However, ArBu showed lower toxicity toward Het-1A human normal esophageal squamous cells, indicating its great selectivity between cancer and normal cells. Moreover, ArBu effectively induced ESCC cell apoptosis mainly by triggering caspase activation through intrinsic and extrinsic pathways. Treatment of ESCC cells also significantly activated p53 signaling by enhancing its phosphorylation. Interestingly, transfection of cells with p53 small interfering RNA significantly inhibited the ArBu-induced p53 phosphorylation and the overall apoptotic cell death. Furthermore, ArBu also demonstrated novel in vivo anticancer efficacy by inhibiting the tumor growth through activation of p53 pathway. Taken together, these results demonstrate the p53-targeting therapeutic potential of bufadienolides against ESCC.

Keywords: arenobufagin, apoptosis, esophageal squamous cell carcinoma, p53

\section{Introduction}

Toad venom (venenum bufonis, also called Chan'su) is derived from the dried skin secretions of giant toads (Bufo gargarizans Cantor or Bufo melanostictus Suhneider) and has been widely used for centuries in traditional Chinese medicine alone or in combination with other herbal ingredients. ${ }^{1,2}$ It is evident that amphibians, such as frogs and toads, possess various bioactive substances in their skin. The discovery of diversified biological activities of the skin extracts has encouraged further study and exploitation of novel amphibian biochemicals with cancer therapeutic application potentials. Many studies have shown that frogs and toads possess different bioactive substances in their skin. In traditional Chinese medicine, amphibian skin extract has been used for the treatment of different diseases. Previous studies have demonstrated that the major pharmacological constituents derived from toad skins are hydrosoluble indole alkaloids (bufotenine, bufotenidine, and cinobufotenine) and liposoluble steroidal cardiac glycosides mainly composed of bufadienolides. ${ }^{3,4}$ Bufadienolide-type cardiotonic steroids are a class of C-24 steroids with a characteristic pyrone ring at $\mathrm{C}-17$ and have been identified as major active components and marker compounds 
of venenum bufonis. ${ }^{5}$ Currently, bufadienolides, including bufotalin, bufalin, and resibufogenin, have been found to show potent cytotoxic and growth inhibitory activity against various human cancer cells. ${ }^{6-15}$ For instance, bufalin and cinobufagin could induce HepG2 cell apoptosis via both Fas- and mitochondria-mediated pathways. ${ }^{9}$ Bufalin exerted antitumor effects by inducing cell cycle arrest and triggering apoptosis in pancreatic cancer cells. ${ }^{12}$ These results have supported the future therapeutic potential of bufadienolides in the treatment of human cancers in clinic.

Arenobufagin ( $\mathrm{ArBu}$ ), a natural bufadienolide compound, has been reported as a novel anticancer compound from toad venom. ${ }^{13,16}$ For instance, Zhang et $\mathrm{al}^{13}$ have shown that ArBu effectively induced apoptosis and autophagy in human hepatocellular carcinoma cells through inhibiting PI3K/Akt/ mTOR pathway. Interestingly, Li et $\mathrm{al}^{16}$ also found that $\mathrm{ArBu}$ could inhibit vascular endothelial growth factor-mediated angiogenesis through suppression of vascular endothelial growth factor receptor 2 signaling pathway. These results have demonstrated the application potential of $\mathrm{ArBu}$ in cancer therapy. However, the effects and mechanisms of $\mathrm{ArBu}$ on esophageal squamous cell carcinoma (ESCC) cells remain elusive. Therefore, in this study, the in vitro and in vivo anticancer activities of $\mathrm{ArBu}$ were examined by comparing with bufalin (Bu), and the underlying molecular mechanisms were also elucidated. The results demonstrated that ArBu effectively impedes the cell growth of a panel of five ESCC cells by inducing p53-mediated apoptosis. ArBu also exhibited novel in vivo anticancer efficacy in ESCC nude mice model by induction of apoptotic cell death. Taken together, these findings indicate the therapeutic potential of ArBu against ESCC.

\section{Materials and methods Reagents and materials}

$\mathrm{ArBu}$ and $\mathrm{Bu}$ standards were provided by Tauto Biotech Co., Ltd (Shanghai, People's Republic of China). Other reagents were purchased from Sigma-Aldrich Co. (St Louis, MO, USA).

\section{Cell lines and cell cultures}

Human ESCC cells (Eca-109, EC9706, TE5, Hec2, and TE11) and a nonmalignant human esophageal squamous cell line Het-1A were obtained from American Type Culture Collection (Manassas, VA, USA) and GuangZhou Jennio Biotech Co., Ltd (Guangzhou, People's Republic of China). The cells were cultured and maintained in RPMI 1640 and Dulbecco's Modified Eagle's Medium supplemented with fetal bovine serum (10\%), penicillin (100 units/mL), and streptomycin (50 units $/ \mathrm{mL}$ ) at $37^{\circ} \mathrm{C}$ in a humid incubator with $5 \% \mathrm{CO}_{2}$.

\section{MTT assay}

The effects of $\mathrm{ArBu}$ and $\mathrm{Bu}$ at different concentrations on the cell growth were determined by MTT assay according to a previous method on a microplate spectrophotometer (VERSA max; Molecular Devices LLC, Sunnyvale, CA, USA). ${ }^{17}$ The $\mathrm{IC}_{50}$ values were calculated by generation of a competitive sigmoidal isoplot.

\section{Flow cytometric analysis}

The effects of $\mathrm{ArBu}$ and $\mathrm{Bu}$ on the cell cycle distribution and cell apoptosis were examined by propidium iodide flow cytometric analysis according to previous protocol. ${ }^{18} \mathrm{Cell}$ apoptosis was estimated by quantifying the sub-G1 peak in the cell cycle profile.

\section{TUNEL assay and DAPI staining}

Terminal dexynucleotidyl transferase(TdT)-mediated dUTP nick end labeling (TUNEL)-4',6-diamidino-2-phenylindole (DAPI) co-staining assay kit (Hoffman-La Roche Ltd., Basel, Switzerland) was employed to detect the apoptotic cell death induced by ArBu. ${ }^{19}$ Briefly, the cells cultured in confocal dish were fixed with $3.7 \%$ formaldehyde for 10 minutes and permeabilized with $0.1 \%$ Triton X-100 in phosphate-buffered saline (PBS). After then, the cells were incubated with $100 \mu \mathrm{L} /$ well TUNEL reaction mixture containing nucleotide mixture and terminal deoxynucleotidyl transferase for 1 hour and $1 \mu \mathrm{g} / \mathrm{mL}$ of DAPI for 15 minutes at $37^{\circ} \mathrm{C}$, respectively. The cells were then washed with PBS and examined under a fluorescence microscope.

\section{Determination of the caspase activity}

The enzymatic activities of caspase-3, -8, and -9 in cells treated with $\mathrm{ArBu}$ were monitored by fluorometric method using specific caspase substrates (Ac-DEVD-AFC for caspase-3, Ac-IETD-AFC for caspase-8, and Ac-LEHD-AFC for caspase-9). ${ }^{20}$

\section{Western blot analysis}

The cells after being treated differently were harvested and collected as cell pellets, which were lysed in lysis buffer (Cell Signaling Technology, Inc., Danvers, MA, USA) on ice for 1 hour. The protein concentration was determined by bicinchoninic acid assay (Sigma-Aldrich Co.). ${ }^{21}$ Equal proteins of each treatment were separated on $12 \%$ sodium dodecyl sulfate 
denaturing polyacrylamide gel and electrophoretically transferred to polyvinylidene fluoride membranes. After blocking with $5 \%$ nonfat milk, the membranes were incubated with primary antibodies (Cell Signaling Technology, Inc.) at 1:1,000 dilution overnight at $4^{\circ} \mathrm{C}$. After washing, the membranes were incubated with corresponding secondary antibodies and visualized by Pierce ECL Western blotting substrate.

\section{siRNA transfection}

Cells were seeded at $2 \times 10^{5}$ cells per well in six-well plates and allowed to grow to $50 \%$ confluence after 24 hours. The cells were then incubated with $50 \mathrm{nmol} / \mathrm{L}$ of p53 small interfering RNA (siRNA) and transfection reagent in serumfree culture medium for 24 hours, and then $1 \mathrm{~mL}$ of fresh completed medium was added to each well for 24 hours with or without $\mathrm{ArBu}$. Additionally, the cells were also transfected with a fluorescein-labeled nontargeted control siRNA, which allows us to monitor the efficiency of transfection.

\section{Immunofluorescence}

Immunofluorescence imaging of phosphorylated p53 was analyzed by immunofluorescence. In brief, cells after treatments were washed with PBS and fixed with 3.7\% formaldehyde in PBS for 15 minutes. After rinsing several times with PBS, the cells were permeabilized with $0.2 \%$ Triton $\mathrm{X}-100$ in PBS at room temperature for 5 minutes. The permeabilized cells were then blocked with $0.1 \%$ bovine serum albumin and subsequently incubated with primary antibody of p-p53 (1:200) overnight at $4^{\circ} \mathrm{C}$, followed by Alexa-488labeled anti-rabbit IgG antibody (1:250) for 1 hour at room temperature. Nuclei were stained with DAPI for 15 minutes after secondary antibody incubation. After washing with PBS, the slides were analyzed using a fluorescence microscopy.

\section{Tumor xenograft in nude mice}

Eca-109 cells $\left(5 \times 10^{6}\right)$ suspended in $200 \mu \mathrm{L}$ PBS were injected subcutaneously into the right lower hind flank of each 6-week-old male nude mouse. The mice were randomly assigned into three groups with five mice in each group. After 10 days, $\mathrm{ArBu}$ dissolved in the solution $\left(\mathrm{DMF}_{\mathrm{v}}: \mathrm{Tween}-80_{\mathrm{v}}\right.$ : saline $\left._{\mathrm{v}}=10: 2: 88\right)$ was given intraperitoneally $(2 \mathrm{mg} / \mathrm{kg}$ and $4 \mathrm{mg} / \mathrm{kg}$ body weight every other day) for 20 days. Control mice received an equal volume of the vehicle (saline) only. Body weights and tumor volumes were monitored in every 2 days. Tumor xenografts were removed and weighed at the end of the experiments (day 20). Part of each tumor tissue was then subjected to further biochemical analysis.
Tumor dimensions were measured with calipers, and the volume was calculated using the formula: volume $=l \times w^{2} / 2$, where $l$ is the maximal length and $w$ is the width. A portion of the tumors from control and treated groups were used for the preparation of tumor lysate and the rest were fixed in $10 \%$ formalin, dehydrated with graded ethanols, and embedded with paraffin for hematoxylin and eosin staining and immunohistochemical analyses. All animal experiments were approved by the Animal Experimentation Ethic Committee of Guangdong No 2 Provincial People's Hospital, the study followed the Companion Animal Care Guidelines.

\section{Immunohistochemical and immunofluorescence staining analysis}

Glutaraldehyde-fixed tumor specimens were embedded with paraffin and cut into $5 \mu \mathrm{m}$ thin sections. Each tissue section was deparaffinized and underwent hematoxylin and eosin and immunofluorescence staining analysis, respectively. Immunofluorescence staining analysis was performed with anti-p-p53 (Ser $\left.{ }^{15}\right)$ and Ki-67 antibodies (1:100) and Alexa Fluor 488-conjugated anti-rabbit IgG antibody (1:100).

\section{Statistical analysis}

In this study, all experiments were performed at least in triplicate, and the data were presented as mean \pm standard error. Difference between the two groups was analyzed by two-tailed Student's $t$-test and that among three or more groups was analyzed by one-way analysis of variance multiple comparisons. Difference with $P<0.05$ (*) or $P<0.01$ (**) was considered statistically significant.

\section{Results and discussion ArBu and Bu effectively inhibit ESCC cell growth}

The in vitro antiproliferative activities of $\mathrm{ArBu}$ and $\mathrm{Bu}$ (Figure 1A) were first screened against five human ESCC cancer cell lines (Eca-109, EC9706, TE5, Hec2, and TE11) and a human esophageal squamous cell line Het-1A. After 72 hours treatment with $\mathrm{ArBu}$ and $\mathrm{Bu}$, cell viability was detected by MTT assay. As shown in Figure 1B, the results of $\mathrm{IC}_{50}$ value revealed that $\mathrm{ArBu}$ and Bu effectively inhibited the cell growth of ESCC cells. ArBu demonstrated higher anticancer efficacy, with $\mathrm{IC}_{50}$ value found at $0.8 \mu \mathrm{M}, 1.2 \mu \mathrm{M}$, $3.2 \mu \mathrm{M}, 2.4 \mu \mathrm{M}$, and $3.6 \mu \mathrm{M}$ for the tested ESCC cells, which was more effective than those of $\mathrm{Bu}$, with $\mathrm{IC}_{50}$ value found at $1.0 \mu \mathrm{M}, 1.0 \mu \mathrm{M}, 2.6 \mu \mathrm{M}, 3.8 \mu \mathrm{M}$, and $4.9 \mu \mathrm{M}$, respectively. In contrast, $\mathrm{ArBu}$ and $\mathrm{Bu}$ showed lower toxicity toward Het-1A human normal esophageal squamous cells, with 

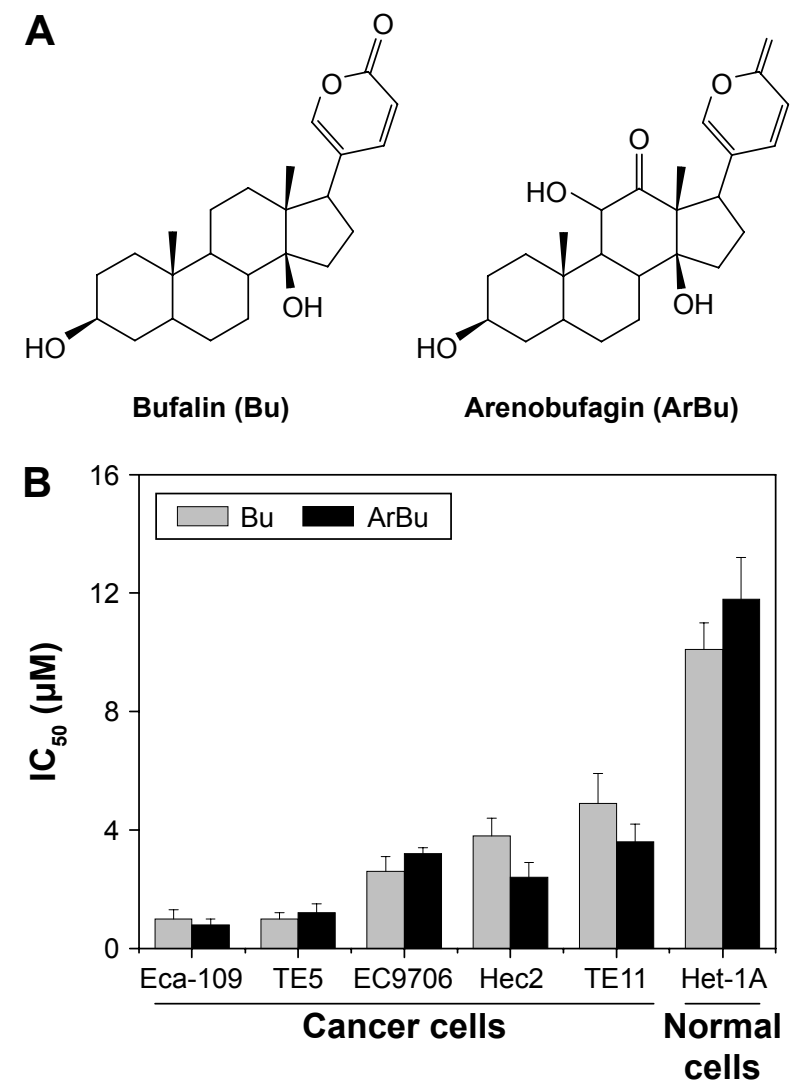

Figure I In vitro anticancer activities of $\mathrm{Bu}$ and $\mathrm{ArBu}$.

Notes: (A) Chemical structure of Bu and $\mathrm{ArBu}$. (B) Cytotoxicity of $\mathrm{Bu}$ and $\mathrm{ArBu}$ toward human cancer and normal cells. The ESCC cells and Het-IA human esophageal squamous cells were treated with $\mathrm{Bu}$ and $\mathrm{ArBu}$ for 72 hours, and the cell viability was examined by MTT assay.

Abbreviations: $\mathrm{Bu}$, bufalin; $\mathrm{ArBu}$, arenobufagin; ESCC, esophageal squamous cell carcinoma.

$\mathrm{IC}_{50}$ value found at $11.8 \mu \mathrm{M}$ and $10.1 \mu \mathrm{M}$ for $\mathrm{ArBu}$ and $\mathrm{Bu}$, respectively, indicating their great selectivity between human cancer and normal cells. Taken together, these results exhibit the therapeutic potential of $\mathrm{ArBu}$ in ESCC treatment.

\section{Activation of cell apoptosis by $\mathrm{ArBu}$}

Studies have shown that the loss of control of cell apoptosis played an important role in carcinogenesis of human cancers. $^{22}$ Therefore, induction of cancer cell apoptosis has been regarded as an effective way to treat cancers. ${ }^{23}$ Many studies have found that most anticancer drugs exhibited their anticancer activities through activating cell apoptosis. ${ }^{24}$ Based on the in vitro anticancer screening, ArBu was found to demonstrate higher anticancer efficacy than $\mathrm{Bu}$. Therefore, studies were also carried out to explore the anticancer mechanism through which ArBu caused cancer cell death. As shown in Figure 2, from the results of TUNEL-DAPI co-staining assay, exposure of Eca-109 cells to $2 \mu \mathrm{M}$ and $4 \mu \mathrm{M}$ of $\mathrm{ArBu}$ for 24 hours triggered significant and dose-dependent

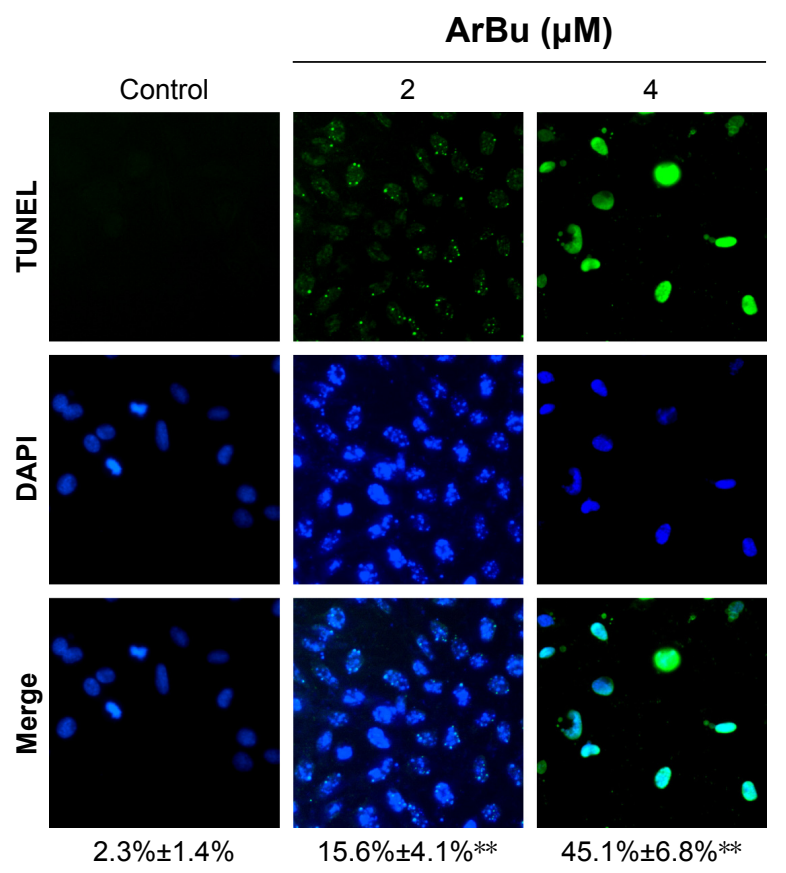

Figure 2 Induction of Eca- 109 cell apoptosis by $\mathrm{ArBu}$.

Notes: Representative images of DNA fragmentation and nuclear condensation as examined by TUNEL-DAPI co-staining assay (magnification, 200x). The cells were treated with $\mathrm{ArBu}$ at $2 \mu \mathrm{M}$ and $4 \mu \mathrm{M}$ for 24 hours. Values in the images indicate the percentage of cell apoptosis induced by $\mathrm{ArBu}$. Difference with $P<0.01$ (**) was considered statistically significant.

Abbreviations: ArBu, arenobufagin; TUNEL, terminal deoxynucleotidyl transferase dUTP nick end labeling; DAPI, 4',6-diamidino-2-phenylindole.

apoptosis. The representative fluorescent images displayed that Eca-109 cells treated with ArBu appeared evident DNA fragmentation and nuclear condensation.

Caspases family proteases act as important factors in regulation of cancer cell apoptosis. ${ }^{25}$ Activated caspases subsequently induced proteolytic cleavage of poly ADPribose polymerase and finally resulted in cell apoptosis. ${ }^{25}$ Cell apoptosis can be initiated by two central mechanisms, the extrinsic (death receptor-mediated) and the intrinsic (mitochondrial-mediated) apoptotic pathways. In this study, we also examined the intracellular caspase activities in cells exposed to $\mathrm{ArBu}$ to examine the requirement of caspases for the apoptotic program. As shown in Figure 3, exposure of Eca-109 cells to ArBu significantly induced the activation of caspase-3, caspase-8, and caspase-9. These results revealed that $\mathrm{ArBu}$ could induce ESCC cell apoptosis mainly through activation of caspase-mediated apoptosis.

\section{Contribution of $\mathrm{p} 53$ pathway to cell apoptosis induced by $\mathrm{ArBu}$}

Tumor suppressor gene p53 acts as an important effector to activate cell apoptosis. Therefore, dysregulation of this process can promote tumor progression and chemoresistance 


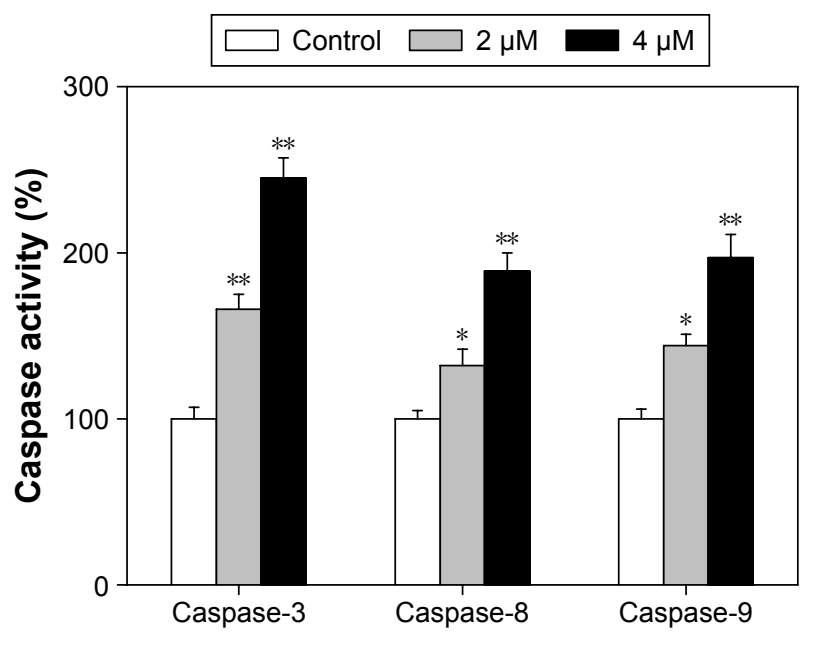

Figure 3 Caspase activities of Eca-109 cells exposed to ArBu as examined using specific fluorogenic substrate.

Notes: $* P<0.05 ; * * P<0.0$ l versus the control. The cells were treated with $\mathrm{ArBu}$ at $2 \mu \mathrm{M}$ and $4 \mu \mathrm{M}$ for 24 hours.

Abbreviation: $\mathrm{ArBu}$, arenobufagin.

during cancer therapy. ${ }^{24,26}$ Therefore, in this study, the levels of total p53 and phosphorylated p53 were examined by Western blot analysis and fluorescence imaging to elucidate the action mechanisms of ArBu. As shown in Figure 4A, $\mathrm{ArBu}$ moderately increased the expression of total p53 protein and significant increase in phosphorylated p-p53 at Ser15 was detected in a dose-dependent manner in Eca109 cells. Phosphorylation of p53 was further confirmed by fluorescence imaging. As shown in Figure 4B, significant increase in green fluorescence intensity in Eca-109 cells demonstrated that the phosphorylation of $\mathrm{p} 53$ protein was induced by $\mathrm{ArBu}$. The role of p53 in ArBu-induced ESCC cell apoptosis was further verified by using RNA interference to silence p53 gene. As shown in Figure 5A, transfection of cells with p53 siRNA significantly inhibited the ArBuinduced $\mathrm{p} 53$ phosphorylation and the overall cell apoptosis by measuring the sub-G1 cell population (Figure 5B), whereas transfection with the negative control siRNA showed no effects in the earlier events. These results suggest that $\mathrm{ArBu}$ could activate ESCC cell apoptosis through activation of p53-dependent pathway.

\section{In vivo anticancer activities of ArBu with involvement of $\mathrm{p} 53$ phosphorylation}

Furthermore, the in vivo anti-ESCC activity of ArBu was evaluated in Eca-109 xenografts in a nude mice model. After being given $\mathrm{ArBu}$ intraperitoneally $(2 \mathrm{mg} / \mathrm{kg}$ and $4 \mathrm{mg} / \mathrm{kg}$ body weight every other day) for 20 days, the nude mice were sacrificed and the tumors were collected for further analysis. The results showed that the tumor weight in nude mice was significantly reduced from $2.3 \mathrm{~g}$ to $1.4 \mathrm{~g}$ and $1 \mathrm{~g}$, respectively. The tumor volume was dramatically decreased to $68 \%$ and $46 \%$ of control group (Figure $6 \mathrm{~A}$ and B). Moreover, the body weight of the mice that had received ArBu treatment maintained constant throughout the treatment process. These results demonstrate the novel in vivo anticancer efficacy of ArBu against ESCC. Besides, immunostaining of histological sections was also conducted to examine the in vivo anticancer mechanisms. As shown in Figure 6C, significant decrease in blood vessel and cancer cell density was observed in tumors treated with $2 \mathrm{mg} / \mathrm{kg}$ and $4 \mathrm{mg} / \mathrm{kg} \mathrm{ArBu}$. The expression of Ki-67, a biomarker of proliferation, was significantly inhibited by $\mathrm{ArBu}$. Moreover, the expression of phosphorylated p53
A

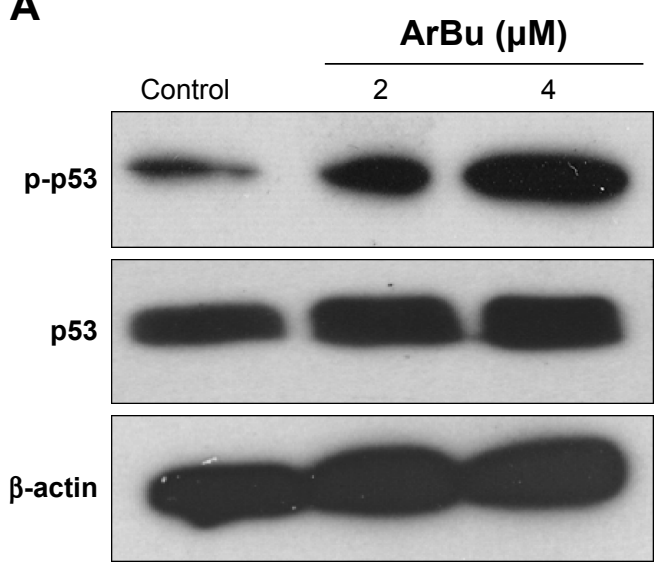

B
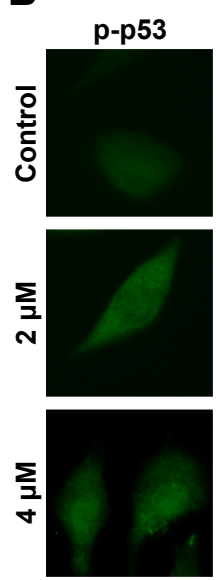
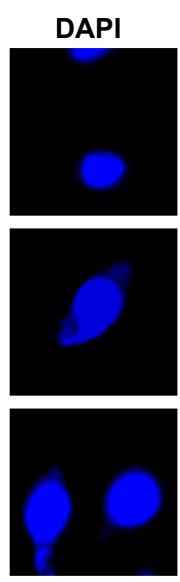

Phase
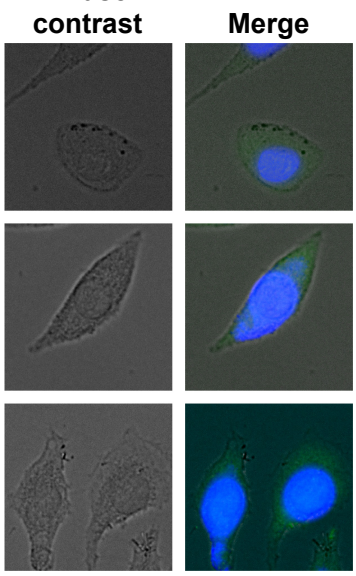

Figure 4 Activation of $\mathrm{p} 53$ by $\mathrm{ArBu}$.

Notes: (A) Phosphorylation of p53 at the sites of Ser 15 and expression of total p53 in Eca- 109 cells induced by ArBu for 24 hours. (B) Immunofluorescence of p-p53 in cells treated with ArBu for 24 hours (magnification, 600x).

Abbreviations: ArBu, arenobufagin; DAPI, 4',6-diamidino-2-phenylindole. 
A

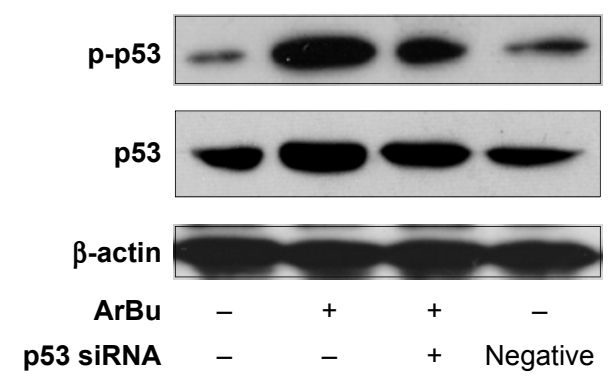

B

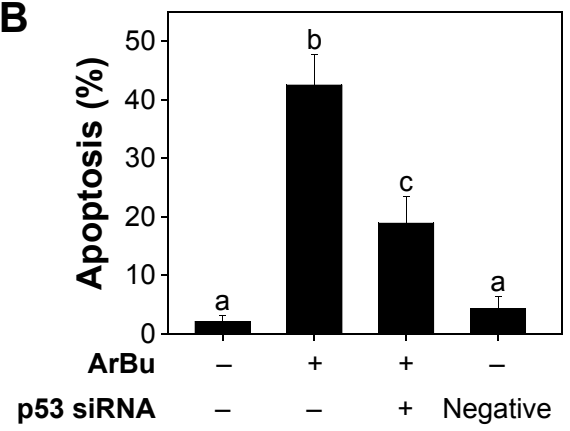

Figure 5 Downregulation of $\mathrm{p} 53$ by siRNA transfection $(\mathbf{A})$ and the effects on cell apoptosis induced by ArBu ( $4 \mu \mathrm{M}, 24$ hours) as examined by PI flow cytometric analysis (B). Notes: All results were obtained from three independent experiments. Bars with different characters $(a, b$, and $c)$ are statistically different at $P<0.05$ level. Abbreviations: siRNA, small interfering RNA; $\mathrm{ArBu}$, arenobufagin; $\mathrm{PI}$, propidium iodide.

was also greatly enhanced after treatment. Furthermore, we have also used TUNEL analysis to confirm the induction of cell apoptosis in the tumor in vivo by ArBu. Therefore, these in vivo data support that $\mathrm{ArBu}$ could inhibit tumor growth through activation of p53-mediated cell apoptosis.

\section{Conclusion}

In this study, the in vitro and in vivo anticancer activities of two bufadienolides (including $\mathrm{ArBu}$ and $\mathrm{Bu}$ ) were examined, and the action mechanisms were also elucidated. The results demonstrated that $\mathrm{ArBu}$ and $\mathrm{Bu}$ could effectively inhibit the cell growth of a panel of five ESCC cells through induction of cell apoptosis through activation of p53 signaling pathway. Besides, ArBu also demonstrated novel in vivo anticancer efficacy in nude mice model, where ArBu remarkably suppressed the tumor growth through activation of p53 pathway. Considering all, these results illustrate the therapeutic potential of bufadienolides against ESCC by regulating p53 pathway.
A

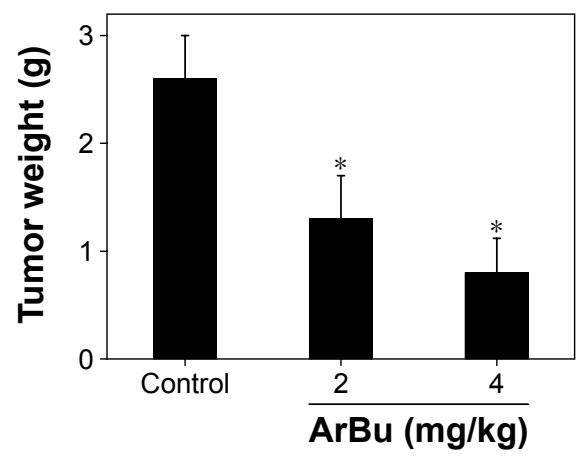

B

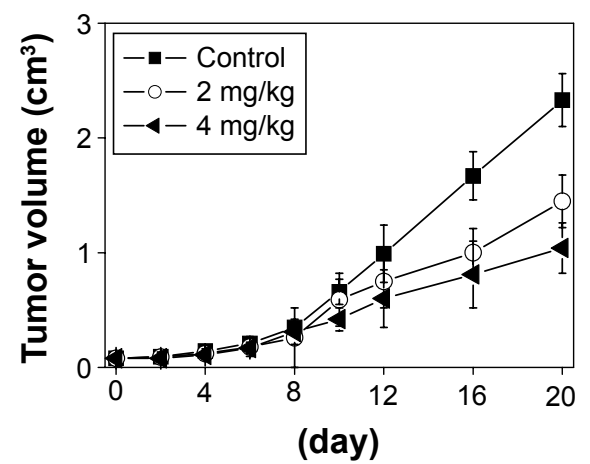

C

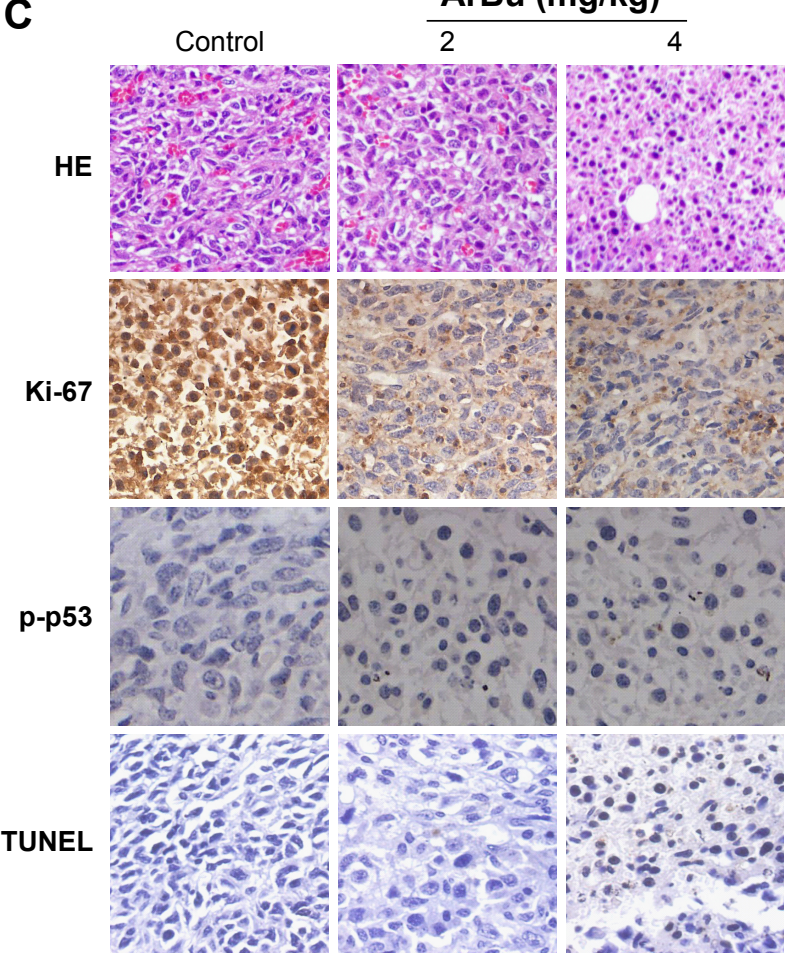

Figure 6 In vivo antitumor efficacy and action mechanisms of ArBu.

Notes: (A, B) Inhibition of Eca- 109 xenograft tumor weight and volume by ArBu $(2 \mathrm{mg} / \mathrm{kg}$ or $4 \mathrm{mg} / \mathrm{kg})$ for 20 days. $* P<0.01$ versus the control. (C) HE staining and immunohistochemical analysis of the expression levels of Ki-67, p-p53, and TUNEL in Eca-109 xenograft tumor treated with ArBu for 20 days.

Abbreviations: ArBu, arenobufagin; HE, hematoxylin and eosin; TUNEL, terminal deoxynucleotidyl transferase dUTP nick end labeling. 


\section{Acknowledgment}

This work was completed with the support of The Construction of Scientific Medicine Research Project of Guangdong Province (2013 20131105).

\section{Disclosure}

The authors report no conflicts of interest in this work.

\section{References}

1. Lu CX, Nan KJ, Lei Y. Agents from amphibians with anticancer properties. Anticancer Drugs. 2008;19(10):931-939.

2. Oelkrug C, Hartke M, Schubert A. Mode of action of anticancer peptides (ACPs) from amphibian origin. Anticancer Res. 2015;35(2):635-643.

3. Qi FH, Li AY, Lv H, et al. Apoptosis-inducing effect of cinobufacini, Bufo bufo gargarizans Cantor skin extract, on human hepatoma cell line BEL-7402. Drug Discov Ther. 2008;2(6):339-343.

4. Liu C, Cao W, Chen Y, Qu D, Zhou J. Comparison of toad skins Bufo bufo gargarizans Cantor from different regions for their active constituents content and cytotoxic activity on lung carcinoma cell lines. Pharmacogn Mag. 2014;10(39):207-212.

5. Huang H, Yang Y, Lv C, et al. Pharmacokinetics and tissue distribution of five bufadienolides from the Shexiang Baoxin Pill following oral administration to mice. J Ethnopharmacol. 2015;161:175-185.

6. Wang Z, Wen J, Zhang J, Ye M, Guo D. Simultaneous determination of four bufadienolides in human liver by high-performance liquid chromatography. Biomed Chromatogr. 2004;18(5):318-322.

7. Wen L, Huang Y, Xie X, et al. Anti-inflammatory and antinociceptive activities of bufalin in rodents. Mediators Inflamm. 2014;2014:171839.

8. Zhai XF, Fang FF, Liu Q, Meng YB, Guo YY, Chen Z. MiR-181a contributes to bufalin-induced apoptosis in PC-3 prostate cancer cells. BMC Complement Altern Med. 2013;13:325.

9. Qi F, Inagaki Y, Gao B, et al. Bufalin and cinobufagin induce apoptosis of human hepatocellular carcinoma cells via Fas- and mitochondriamediated pathways. Cancer Sci. 2011;102(5):951-958.

10. Chen YY, Lu HF, Hsu SC, et al. Bufalin inhibits migration and invasion in human hepatocellular carcinoma SK-Hep1 cells through the inhibitions of NF-kB and matrix metalloproteinase-2/-9-signaling pathways. Environ Toxicol. 2015;30(1):74-82.

11. Wang W, Shi A, Fan Z. Apoptosis of T-47D cells induced by cinobufacini via a caspase-3-dependent manner. Chem Res Chin Univ. 2014; 30(1):108.

12. Li M, Yu X, Guo H, et al. Bufalin exerts antitumor effects by inducing cell cycle arrest and triggering apoptosis in pancreatic cancer cells. Tumour Biol. 2014;35(3):2461-2471.
13. Zhang DM, Liu JS, Deng LJ, et al. Arenobufagin, a natural bufadienolide from toad venom, induces apoptosis and autophagy in human hepatocellular carcinoma cells through inhibition of PI3K/Akt/mTOR pathway. Carcinogenesis. 2013;34(6):1331-1342.

14. Xu GX, Wang TT. Apoptosis of lens epithelial cells induced by cinobufagin in vitro. Int J Ophthalmol. 2010;3(2):128-131.

15. Li BJ, Tian HY, Zhang DM, et al. Bufadienolides with cytotoxic activity from the skins of Bufo bufo gargarizans. Fitoterapia. 2015; 105:7-15.

16. Li M, Wu S, Liu Z, et al. Arenobufagin, a bufadienolide compound from toad venom, inhibits VEGF-mediated angiogenesis through suppression of VEGFR-2 signaling pathway. Biochem Pharmacol. 2012;83(9): 1251-1260.

17. Luo H, Wang F, Bai Y, Chen T, Zheng W. Selenium nanoparticles inhibit the growth of HeLa and MDA-MB-231 cells through induction of S phase arrest. Colloids Surf B Biointerfaces. 2012;94:304-308.

18. Liu S, Cao W, Yu L, et al. Zinc(II) complexes containing bis-benzimidazole derivatives as a new class of apoptosis inducers that trigger DNA damage-mediated p53 phosphorylation in cancer cells. Dalton Trans. 2013;42(16):5932-5940.

19. Liu W, Li XL, Wong YS, et al. Selenium nanoparticles as a carrier of 5-fluorouracil to achieve anticancer synergism. ACS Nano. 2012; 6(8):6578-6591.

20. Li XL, Chen T, Wong YS, et al. Involvement of mitochondrial dysfunction in human islet amyloid polypeptide-induced apoptosis in INS-1E pancreatic beta cells: an effect attenuated by phycocyanin. Int J Biochem Cell Biol. 2011;43(4):525-534.

21. Zhang Y, Li X, Huang Z, Zheng W, Fan C, Chen T. Enhancement of cell permeabilization apoptosis-inducing activity of selenium nanoparticles by ATP surface decoration. Nanomedicine. 2013;9(1):74-84.

22. Maiese K, Chong ZZ, Shang YC, Wang S. Targeting disease through novel pathways of apoptosis and autophagy. Expert Opin Ther Targets. 2012;16(12):1203-1214.

23. Chen T, Wong YS. Selenocystine induces apoptosis of A375 human melanoma cells by activating ROS-mediated mitochondrial pathway and p53 phosphorylation. Cell Mol Life Sci. 2008;65(17):2763-2775.

24. Chen T, Wong YS. Selenocystine induces caspase-independent apoptosis in MCF-7 human breast carcinoma cells with involvement of p53 phosphorylation and reactive oxygen species generation. Int J Biochem Cell Biol. 2009;41(3):666-676.

25. Riedl SJ, Shi Y. Molecular mechanisms of caspase regulation during apoptosis. Nat Rev Mol Cell Biol. 2004;5(11):897-907.

26. Fridman JS, Lowe SW. Control of apoptosis by p53. Oncogene. 2003;22(56):9030-9040.
OncoTargets and Therapy

\section{Publish your work in this journal}

OncoTargets and Therapy is an international, peer-reviewed, open access journal focusing on the pathological basis of all cancers, potential targets for therapy and treatment protocols employed to improve the management of cancer patients. The journal also focuses on the impact of management programs and new therapeutic agents and protocols on

\section{Dovepress}

patient perspectives such as quality of life, adherence and satisfaction. The manuscript management system is completely online and includes a very quick and fair peer-review system, which is all easy to use. Visit http://www.dovepress.com/testimonials.php to read real quotes from published authors. 